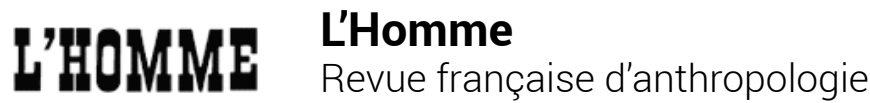

166 | avril-juin 2003

Malinowski, Faulkner. Culture et cognition. Souvenir et héritage

\section{Question d'identité}

L'héritage de la veuve de l'oncle maternel chez les Dì̀ (Nord-Cameroun)

Jean-Claude Muller

\section{OpenEdition}

\section{Journals}

Édition électronique

URL : http://journals.openedition.org//homme/219

DOI : 10.4000//homme.219

ISSN : 1953-8103

Éditeur

Éditions de l'EHESS

Édition imprimée

Date de publication : 30 juin 2003

Pagination : 87-106

ISBN : 2-7132-1805-5

ISSN : 0439-4216

\section{Référence électronique}

Jean-Claude Muller, «Question d'identité », L'Homme [En ligne], 166 | avril-juin 2003, mis en ligne le 08 septembre 2008, consulté le 19 avril 2019. URL : http://journals.openedition.org//homme/219 ; DOI : 10.4000/lhomme.219 


\title{
Question d'identité
}

\author{
L'héritage de la veuve de l'oncle maternel \\ chez les Diì (Nord-Cameroun)
}

Jean-Claude Muller

Q

UELLES SONT les raisons profondes qui permettent à un neveu utérin d'hériter de la veuve de son oncle ? Telle est la question que cet article se propose d'explorer et à laquelle il tentera de répondre - une question à tout le moins embarrassante en anthropologie comparative. Dans un texte fondateur, "The Study of Kinship Systems ", Radcliffe-Brown (1952 : 81) l'avait soulevée sans toutefois en pousser l'examen plus avant. Après avoir remarqué que ce type de mariage pouvait être associé à une terminologie de type crow, un système matrilinéaire classique dans lequel un neveu utérin hérite normalement de son oncle, incluant quelquefois sa veuve, il ajoute :

"But it is also found in many other places where that type of terminology does not exist. Nor is it correlated with matrilineal descent, for it is to be found in African societies that are markedly patrilineal in their institutions. There does not seem to be any theoretical explanation that will apply to all the known instances of this custom. There is no time on this occasion [la présentation orale de l'article par son auteur] to discuss this subject by an analysis of instances. "

C'est à l'examen d'un de ces exemples, les Dì̀, que nous nous attacherons. Cependant, nous l'inclurons, pour consolider notre argument, dans un

Cet article est le résultat d'un travail de terrain de vingt-quatre mois étalé sur dix ans.

Commencé rapidement par une pré-enquête financée par le CAFIR de l'Université de Montréal en été 1990, il se poursuivit ensuite de septembre 1991 à mi-janvier 1992, puis durant les étés 1992, 1993, 1994, 1995, 1996, 1998 et 1999 grâce au fonds CRSH (Canada) et FCAR (Québec) pour les deux derniers séjours. Je remercie Eldridge Mohammadou, alors directeur de l'ancien Institut des sciences humaines à Garoua, de m'avoir signalé les Dì̀ comme terrain digne d'attention et de m'avoir matériellement soutenu, par l'entremise de l'ISH, lors de mes deux premiers séjours. Pour les derniers, j’ai bénéficié de l'aide secrétariale et technique d'Anthropos, un projet conjoint des Universités de Ngaoundéré (Cameroun) et de Tromsø (Norvège). Je remercie aussi vivement mes deux assistants, MM. Adamou Galdima et Oussoumanou Babbawa. Alfred Adler m'a fait d'utiles commentaires sur une première version de ce texte, ce dont je lui sais gré. 


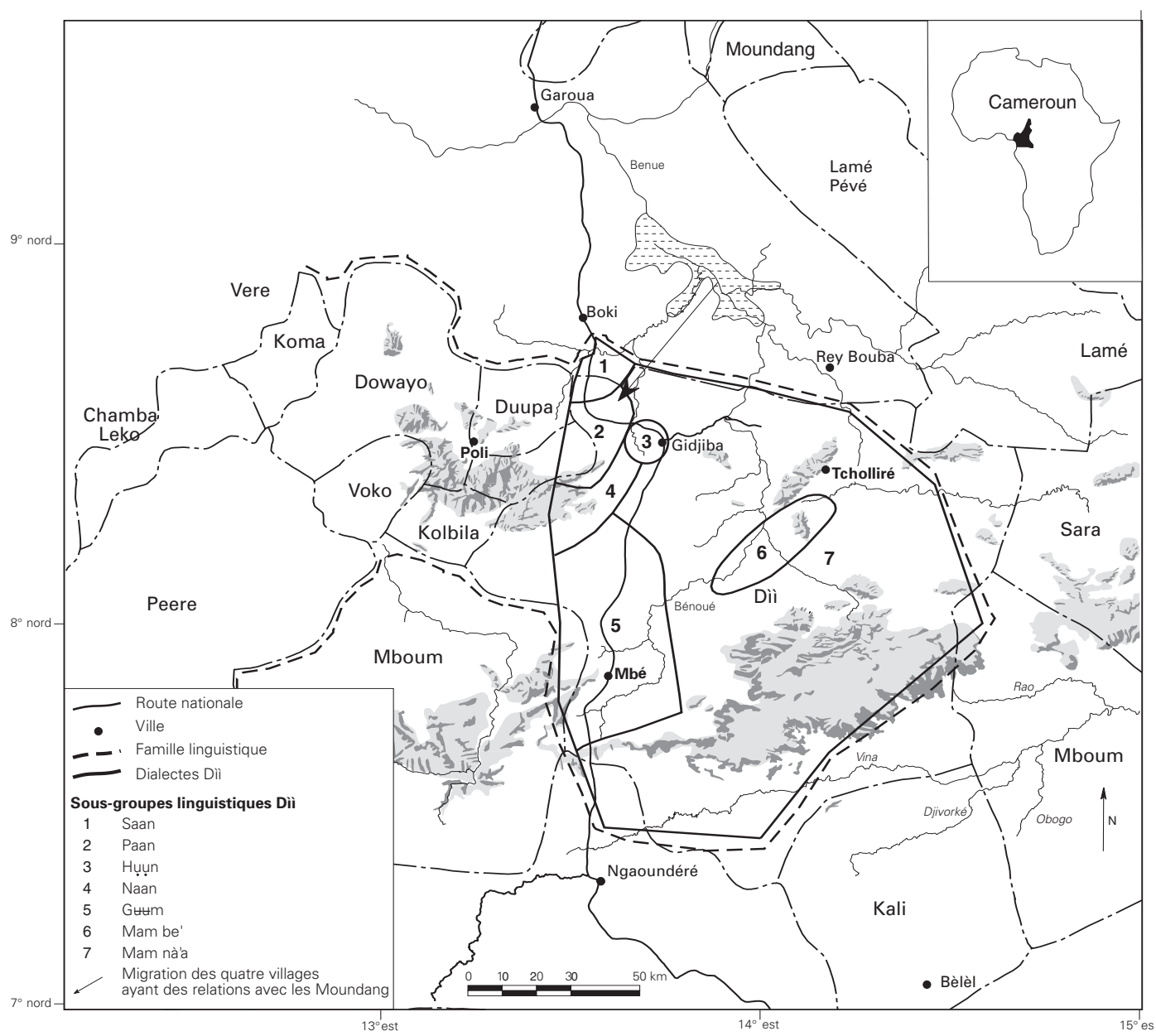

Carte ethnique des Diì et de leurs voisins

ensemble plus vaste comprenant les voisins proches qu'ils connaissent. Nous terminerons brièvement en examinant un cas célèbre qui, à première vue, pourrait sembler contraire mais qui ne fait que renforcer l'argument.

\section{Les Dì̀}

Les Dì̀ vivent dans l'Adamaoua, au Nord-Cameroun. Au nombre de 40 à 50 000, ils sont installés en majorité le long de la route qui va de Garoua à Ngaoundéré, entre le lieu-dit Carrefour Poli et la falaise de Ngaoundéré; au sud de Tcholliré, de Mbé à Sassa Mbersi, dans la cuvette de la Bénoué, ainsi que sur le plateau, au nord-est de l'axe Ngaoundéré-Ganha (voir carte). On distingue plusieurs familles linguistiques qu'on évoquera plus loin (cf. infra p. 96). Les Dì̀ sont groupés en plus d'une centaine de petites chefferies, $k a a$, elles-mêmes structurées autour de quelques lignages patrilinéaires 
tenus comme nécessaires à leur fonctionnement. Cette organisation est décrite le plus souvent au travers de leur cérémonie la plus importante, la circoncision. Le lignage dominant, zig gbanàà, est celui du chef qui est chargé de l'organiser; le lignage pratiquant l'opération, celui du circonciseur, zig dọ̀ nàà, vient en second, et un lignage dit autochtone, zig waa hág, est chargé de préparer et de "balayer " la place de la circoncision (il existe plusieurs de ces lignages autochtones qui se partagent d'autres tâches et dont l'ordre de préséance importe peu). Enfin, pour fabriquer les couteaux, il est nécessaire de faire appel à un lignage de forgerons, zig naๆ, qui forment un groupe endogame. Chez les Dì̀ de dialecte g l'office de circonciseur n'existe pas, le forgeron cumulant les deux rôles. Les chefferies abritent aussi, en nombre variable, des ressortissants d'autres chefferies qui, eux-mêmes ou leurs ancêtres, sont venus y habiter pour de multiples raisons. Ceux-ci sont quelquefois plus nombreux que les résidents des lignages originels. Les Dì̀ sont d'experts agriculteurs, connus au Cameroun pour leur production d'ignames, vendues loin à la ronde. Cependant, ils se définissent comme des gens du mil et du sorgho - et, de plus en plus, du maïs -, céréales qui constituent la base de leur régime alimentaire.

La nomenclature du système de parenté comporte la règle d'obliquité de type crow, normalement associée à des systèmes matrilinéaires - et parfois à double descendance unilinéaire (Muller 1997). Quelques caractéristiques saillantes de ce système seront examinées plus loin car les Dì̀ ont une exégèse élaborée tentant d'expliquer cette règle d'obliquité par l'héritage de la veuve de l'oncle maternel. Mais, contrairement à ce que la terminologie de la parenté pourrait laisser croire, les Dì̀ sont patrilinéaires et patrivirilocaux, bien qu'ils affirment qu'un enfant appartient aussi bien au liz (" côté ») de son père qu'à celui de sa mère. Le suffixe " côté » indique ici toute la parenté cognatique de la mère ou du père, mais plus particulièrement le lignage paternel, zig bà 'á, et le patrilignage, zig nà 'á, d'où sa mère est issue. Cela n'influe en rien sur l'héritage des biens et des offices qui passent de frère aîné à frère cadet ou de père à fils, mais cette double appartenance a une incidence sur les prohibitions du mariage.

Le régime matrimonial des Dì̀ est paradoxal (Muller 1998). Quoique le système terminologique utilise la règle d'obliquité crow, les prohibitions généalogiques sont exprimées comme le seraient celles d'un système de type omaha. Les patrilignages des parents, des grands-parents et des arrièregrands-parents (F, M, FM, MM, MMM, MFM, FMM, FFM) sont interdits ainsi que tous les cognats très proches qui n'appartiennent pas à ces lignages. Les cognats lointains sont eux aussi en théorie interdits mais souffrent de nombreuses exceptions. Quant aux prohibitions affinales, l'échange de « sœurs » entre lignages était totalement proscrit. Il est toujours 
fortement réprouvé, bien que certains lignages modernistes le tolèrent, alors que la reduplication des alliances entre lignages avec des partenaires du même sexes, toujours permise, était autrefois très valorisée. Il s'agit d'une question de statut: le donneur de femme est supérieur au preneur et les deux positions sont perçues comme contradictoires. Dans l'échange de "sœurs", chacun des lignages est à la fois l'un et l'autre, ce qui, selon les Dì̀, est impossible. Les modernistes, qui redupliquent ces alliances avec des germains de sexes différents, ne font que réduire cette opposition en prenant simplement garde que la femme "donnée » et celle "rendue » impliquent deux segments distincts du même lignage. Même s'il y a échange au niveau du lignage, cet échange n'en est pas un pour ce qui concerne les segments, l'important, pour les modernistes, étant que le même homme d'un lignage donné ne puisse être à la fois donneur et preneur. Dans le cas de la réplication de l'alliance avec des germains de même sexe, ce problème du statut ne se pose pas car il ne change pour aucun des protagonistes. C'est dans ce contexte matrimonial que la maxime qui énonce qu'un enfant appartient aussi bien au lignage, ou "côté " de son père, qu'à celui de sa mère devient opératoire. Les hommes d'un lignage n'épouseront pas les filles des sœurs du lignage qui leur a donné une épouse. Ce serait un échange différé puisque ces filles appartiennent, selon la règle, aussi bien au lignage de leur père qu'à celui de leur mère où le lignage donneur en question a déjà fourni une fille. On peut toujours continuer à donner des femmes au même lignage, mais on ne peut en recevoir de lui.

Tout mariage demande un prix de la fiancée. Celui-ci valide le mariage et nous nous y attarderons car il constitue l'une des justifications de l'héritage de la veuve de l'oncle maternel. Il existe plusieurs façons d'obtenir une épouse dont le point commun est d'inclure un prix de la fiancée légitimant l'union et affiliant les enfants au père. Cependant, l'argument principal des Dì̀ pour expliquer l'héritage de la veuve réside dans la règle d'obliquité crow de leur système terminologique. Selon cette règle, les enfants de l'oncle maternel sont les «enfants » d'Ego, et les enfants de la tante paternelle sont ses "pères » et " mères ». Pour les Dì̀, cette terminologie anticipe le statut d'Ego qui deviendra "père " adoptif des enfants de sa future femme lorsqu'il héritera de la veuve de son oncle ou lorsque sa mère sera héritée par le neveu utérin de son mari pour lequel Ego deviendra un «enfant » adoptif. Les Dì̀ raisonnent exactement comme l'ont fait plusieurs ethnologues célèbres, dont la liste est donnée par Murdock (1949 : 123), en particulier par Rivers et par Lowie. Les Dì̀, et ces illustres prédécesseurs, font découler cette règle d'obliquité, qu'elle soit crow ou omaha dans le cas inverse, d'un type de mariage dérivé. Mais il peut être démontré que ces règles d'obliquité n'ont le plus souvent rien à voir avec 
l'héritage des veuves et qu'il s'agit de tout autre chose (Muller 1997). Même si quelques cas assez rares, comme celui des Dì̀, montrent cette corrélation, elle n'en est pas une de cause à effet mais une simple coïncidence, ce que Radcliffe-Brown avait bien relevé.

Le mariage, lui aussi, justifie l'héritage, fẹe kéé éé, "il hérite, reprend femme mariée ", de la veuve de l'oncle mais également celui de la veuve d'un frère aîné par son cadet, les protagonistes s'appelant déjà mutuellement en plaisantant "mon épouse ", kẹẹ mí, et « mon mari », wạa mí, dans les deux situations. Le second cas s'explique routinièrement comme un héritage agnatique - ce sont les frères cadets qui héritent de leurs aînés. Cependant, le premier donne lieu à des explications qui peuvent nous sembler des plus controuvées, comme si, pour les Dì̀, le second allait de soi. Ces explications rejoignent aussi la théorie de l'échange généralisé élargi telle que l'a formulée Claude Lévi-Strauss. Fondée sur le prix de la fiancée, cette théorie dit que toute femme est donnée contre un "prix de la fiancée " qui permettra ensuite à son "frère " de l'utiliser pour se procurer à son tour une conjointe. C'est cette théorie qui entraîne les Dì̀ à refuser les mariages par échange entre deux lignages car on ne fait qu'échanger des "prix de la fiancée » deux à deux, alors qu'ils doivent être un médium pour unir plusieurs lignages en un cercle qui se referme sur lui-même. Ethnologues et ethnographiés sont, encore une fois, sur la même longueur d'onde théorique. L'héritage de la veuve de l'oncle est justifié comme suit: "Le "prix de la fiancée" donné pour ma mère a permis à son frère, mon oncle maternel, de se procurer une épouse. Comme il est mort, je puis donc le réclamer ou épouser sa femme ». Mais cela n’est pas tout, car ces arguments vont beaucoup plus loin en employant, pourraiton dire, le second degré, pour justifier cet héritage par le frère cadet de la veuve du frère aîné. Un tel argument s'énonce ainsi : "Si ma mère avait été un homme, elle aurait dû hériter de son frère. Comme elle ne le peut pas, étant femme, c'est moi qui doit la remplacer». On joue ici sur les implicites de la situation : la mère de l'héritier est femme et, dans ce contexte, elle demeure une cadette, assimilée comme telle en toute circonstance. L'héritage est donc possible dans tous les cas puisque, si celui-ci est interdit entre une veuve et un frère aîné du défunt, il reste permis entre la veuve et un frère cadet ou un substitut de frère cadet, ce qu'est toujours la mère de l'héritier qui la remplace. On notera que le même argument est utilisé par certaines populations ghanéenne pour expliquer les "chapardages rituels " du neveu utérin (Goody 1969a: 57-60). Si la mère était née homme, son fils aurait été intégré dans le lignage de sa mère; il rappelle ce fait en chapardant des biens produits par ce lignage car il en fait un peu partie comme membre de ce que Goody appelle résiduel. 
Pour les Dì̀, l'héritage potentiel de la veuve de l'oncle maternel explique les relations de parenté à plaisanteries qui existent entre tous les neveux utérins et la femme de l'oncle maternel, que ces oncles soient des cadets ou des aînés de la mère, peu importe, puisque la mère est toujours cadette, même du plus jeune de ses frères. Cette relation s'appelle yàg fạa fólí ou fóó yàg fạa: «il joue parole belle-sœur»; le neveu ou l'épouse de l'oncle peuvent indifféremment provoquer les plaisanteries, quelquefois scabreuses, et l'oncle n'a rien à dire. Les neveux se vantent souvent en disant que l'oncle quitte la place pour ne pas avoir à entendre les propos échangés qui le mettraient mal à l'aise. Le neveu feint de faire la cour à sa tante par alliance, qu'il appelle kẹé mî́, "ma femme», la réciproque étant wạa mín, "mon mari ", et il peut se permettre des entorses à la bienséance, comme, par exemple, voler de la nourriture préparée par sa tante ou même entrer dans la cuisine, ce qui ne se fait pas normalement et est vu comme une grande incivilité puisqu'aucun homme n'est autorisé à pénétrer dans aucun de ces lieux après sa circoncision. Cette prohibition est même spécifiquement enseignée à la fin de l'opération avec la conséquence de sa rupture: faire repousser le prépuce. Les nièces utérines plaisantent aussi avec l'épouse de leur oncle maternel, et ces plaisanteries se nomment également yàg fạa fóólí. C'est un terme général qui concerne toutes ces relations à libre parler quand elles impliquent deux femmes entre elles ou des hommes et des femmes mais on peut être plus précis : les femmes appellent la relation yàg wạa fóóĺ lorsqu'elle implique le neveu ou un frère cadet de leur mari. Cette relation est spécifique à la femme de l'oncle, elle ne se confond pas avec le chapardage rituel si commun dans les sociétés africaines patrilinéaires, qui permet au neveu d'agonir d'injures son oncle maternel et de lui voler de la viande ou des pots de bière. Les Dì̀, au contraire, plaisantent peu avec lui mais beaucoup avec sa femme. La relation avec l'oncle est ainsi faite de complicité et d'attitudes très détendues. À la question : "Que peut-on attendre de son oncle maternel ? ", bien des Dì̀ répondent, de façon caractéristique, que le neveu peut toujours aller s'établir chez lui sans problèmes, ce qu'ils font souvent sans toutefois abandonner leur affiliation lignagère patrilinéaire d'origine.

Nous avons vu que c'est cet héritage potentiel qui pousse les Dì̀ à justifier leur règle d'obliquité crow. Comme cet héritage est largement chose du passé - bien que passé récent -, les Dì̀, ayant le plus l'esprit sociologique, sont d'avis qu'il faut changer certaines caractéristiques de leur système terminologique de parenté. Ce sont les termes désignant les cousins croisés qui sont visés et la réforme préconisée est de les aligner sur ceux utilisés pour les cousins parallèles, qui sont des «frères " et des «sœurs ", transformant leur système en type hawaïen pour les cousins. Il faut aussi 
ajouter que ces cas d'héritage étaient plutôt rares et plus théoriques que réels, la différence d'âge entre oncle et neveu étant en général assez grande. $\mathrm{Si}$, toutefois, l'épouse de l'oncle était jeune, elle était aussi convoitée par les frères cadets du défunt qui avaient plus de chance de lui agréer, surtout si la veuve avait des enfants qui seraient alors élevés dans la maison de leur père défunt ou dans son segment de lignage. Cependant, j'ai recueilli deux cas d'héritage assez récents de la veuve de l'oncle maternel: le premier implique un défunt qui avait deux femmes, ainsi qu'un frère cadet et un neveu utérin : le frère et le neveu prirent chacun une des deux épouses. Le second, tiré des archives du tribunal de l'arrondissement de Mbé, évoque le cas d'un neveu utérin qui vint porter plainte au tribunal parce que le père de l'épouse de son oncle maternel, décédé, l'avait donnée, après sa mort, en mariage à un autre homme, le mari n'ayant pas de frère cadet. Le neveu revendiqua soit la veuve soit le "prix de la fiancée » reçu pour ce second mariage. Le père céda alors sa fille au neveu et remboursa le «prix de la fiancée » au nouveau mari. Dans ces cas d'héritage, les enfants nés du premier mariage restent affiliés à leur père défunt, même s'ils suivent - temporairement - leur mère chez son nouveau conjoint; celui-ci sera le père légal des enfants nés après le remariage.

L'héritage le plus pratiqué est donc celui des veuves du frère aîné, jamais celle du frère cadet, mais seulement si la veuve le désire. L'héritage des veuves par les frères cadets du défunt est une coutume plus générale et plus commune que l'héritage intergénérationel de la veuve de l'oncle maternel ou paternel ou même des femmes du père à l'exclusion de sa propre mère (Murdock 1949: 23-24), et on pourrait penser qu'il aurait mieux valu en traiter d'abord, mais les Dì̀ voient celui de la veuve de l'oncle comme premier, le second étant son dérivé ${ }^{1}$. L'héritage est cependant interdit entre deux frères ou demi-frères paternels qui ont épousé des demi-sœurs paternelles ou utérines, deux sœurs entières, en principe, ne pouvant épouser deux frères ou demi-frères paternels (Muller 1998 : 65-67)². Dans ce cas l'héritier serait le mari de deux sœurs, ce qui est formellement interdit.

Pour bien comprendre l'héritage de la veuve de l'oncle dans le contexte général, il faut encore dire un mot de la grande extension dì̀ des termes

1. L'héritage intergénérationnel des femmes du père est vu comme une grossièreté digne des Toupouri, des Massa, des Moundang et des Dowayo, à qui les Dì̀ créditent, à tort ou à raison, ce genre de perversion. Il a toujours été formellement interdit chez ceux qui parlent les dialectes mam be' et mam nà'a, qui sont la grande majorité; il semble que certains de ceux parlant les dialectes gum, paan, naan et saan l'aient occasionnellement pratiqué il y a longtemps, mais y ont aujourd'hui renoncé.

2. Cela semble bien être relié à l'interdiction de ce que Françoise Héritier (1994) appelle le « redoublement de l'identique ». Les Dì̀, interrogés sur cette possibilité, voient l'affaire sous un angle très matricentré. J'ai eu la surprise de les entendre parler de l'inceste comme de hen yààd, "les choses du chien ", tout comme les Samo l'ont décrit à Françoise Héritier (ibid. : 187), car le chien couche avec sa mère et ses sœurs alors que le cheval, lui, et le seul parmi les animaux, se comporte en ces matières comme un humain .../... 
oncle maternel/neveu utérin. L'un et l'autre s'appellent et se réferent comme pạ. Cette extension est essentielle pour saisir la logique du mariage avec la veuve de l'oncle. Tous les hommes d'un lignage ayant reçu une femme d'un autre désignent les hommes de la génération supérieure de ce lignage comme pạ, et ce réciproquement. Mais l'héritage est interdit entre ces partenaires; malgré l'équivalence linguistiquement exprimée, il n’est permis qu'avec la veuve d'un vrai oncle. Si l'« oncle » classificatoire n'est pas parent généalogique avec son "neveu » classificatoire, celui-ci peut épouser sa fille. La relation cesse alors pour devenir celle de beau-père à gendre. Pour pouvoir prétendre à l'héritage, il faut donc que l'oncle maternel soit préférentiellement un frère entier de la mère. Tous les vrais neveux utérins ont théoriquement droit à la veuve d'un vrai oncle, frère entier de la mère. Les Dì̀ tiennent cependant pour théoriquement possible, mais non attesté, l'héritage de la veuve d'un homme par le fils de sa demi-sœur utérine. Les deux hommes sont en relation terminologique d'oncle et de neveu mais comme le défunt et sa sœur ne sont que demi-germains utérins, le neveu est assez éloigné de ce demi-oncle pour que ses prétentions à l'héritage

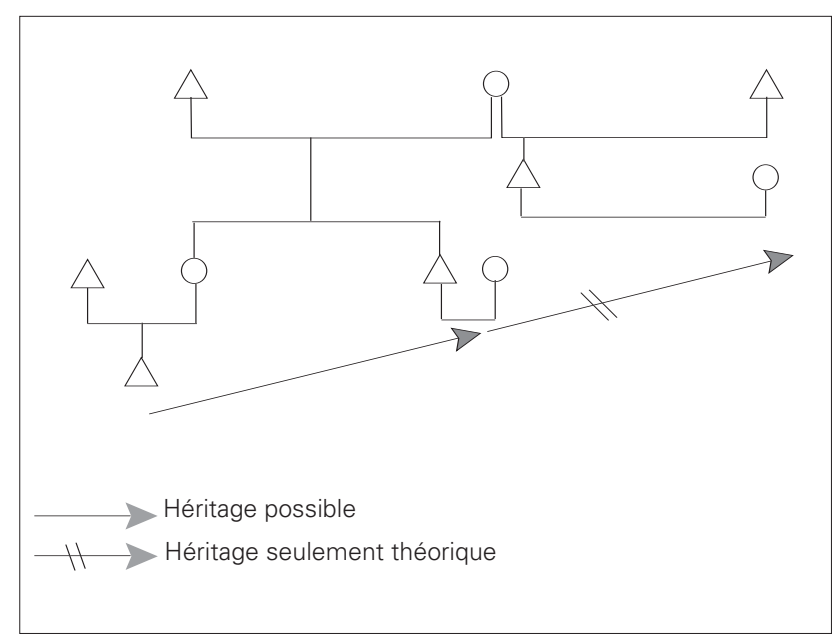

Fig. I soient mises en échec, ce qui explique, pour les Dì̀, qu'on n'en trouve aucun cas (voir Fig. 1). Il en va différemment pour un utérin parallèle qui appelait le défunt " oncle maternel ». Ces cas sont rares, mais j'ai la mention détaillée d'au moins deux exemples de veuves

Je pense que cette opinion sur le cheval doit venir des Peuls; ceux-ci l'ont introduit chez les Dì̀ qui ne le connaissaient pas et qui ne l'ont, en fait, jamais élevé. Cette croyance peut aussi venir des peuples du Logone qui élevaient des poneys en prenant soin de respecter en les appariant leurs propres catégories de l'inceste (Seignobos 1987). Toujours est-il que les Dì̀ établissent une relation d'intensité décroissante entre les incestes. On ne couche ni avec sa mère, sa sœur ou sa fille. Ce sont les pires incestes, tous placés sur le même plan. Ceux de "deuxième type ", moins graves et donnés par ordre décroissant, sont de coucher à la fois avec la mère et la fille, avec deux sœurs entières et, enfin, deux demi-sœurs utérines. Quant aux relations avec deux demi-sœurs paternelles, on les évoque avec un clin d'œil des plus grivois. Une telle relation entre un homme et deux demi-sœurs paternelles non mariées est considérée comme une sorte d'exploit, car il faut éviter que les sœurs le sachent sinon elles seraient jalouses et rompraient. Dans une telle situation, toute hypothétique - je n'ai pas pu recueillir de cas réels -, mais narrée avec un grand sérieux accompagné de sourires en coin, l'accent est mis sur la circonspection de l'amant qui doit éviter de se faire surprendre par une des deux sœurs plutôt que sur le malaise que devrait susciter la relation. 
héritées par le fils de la fille de la sœur utérine de la mère du défunt. Ce parent parallèle hérite donc de la veuve du fils de la sœur utérine de la mère de sa mère (MMZSW). La mère de l'héritier est, d'après le système terminologique dì̀, une «sœur" du décédé que l'héritier appelait "oncle maternel ». Le droit d'héritage passe ainsi à la génération suivante pourvu qu'il soit transmis par deux sœurs utérines. Ce ne sont pas les lignages patrilinéaires qui sont ici en cause - bien qu'ils aient la priorité pour l'héritage mais la composante matrilinéaire de la parenté (voir Fig. 2).

En dehors des cas particuliers où l'héritage entre frères ne peut se faire, un homme et ses sœurs cadettes plaisantent avec toutes les femmes de tous

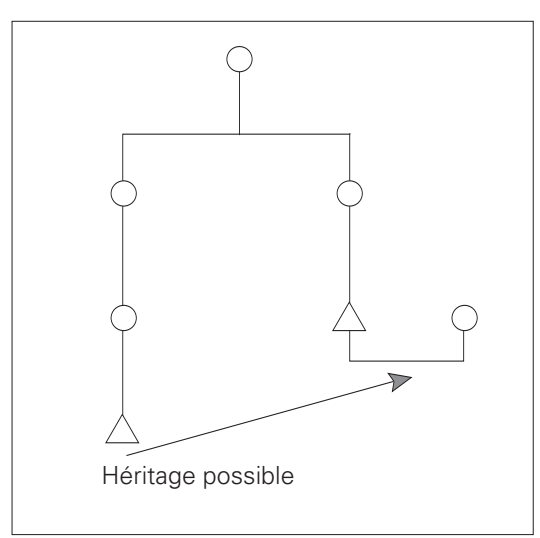

Fig. 2 leurs frères aînés de lignage et de tous les cognats que le frère aîné appelle "frères cadets ». L'expression est la même, fóó yàg fạa ou fóó yạg wạa, que celle utilisée pour caractériser les relations avec l'épouse de l'oncle maternel. On couche quelquefois en cachette avec la femme d'un frère aîné de lignage, même si cela est interdit, et au risque de se faire sévèrement corriger par le mari trompé. Celui-ci ne peut aller se plaindre à la cour de justice car cette action n'est pas considérée comme un adultère, kẹé bèèl', "femme appeler». Le mari va tempêter mais ne fera rien sinon corriger le coupable; c'est une affaire qui reste en famille et n'en sort pas. Du moins, essaie-t-on... Il est cependant interdit à un frère de coucher avec la femme de son frère aîné entier - et avec tout autre femme avec laquelle il aurait couché - sous peine de la sanction suivante: si l'un des deux frères voit l'autre malade, ce dernier meurt. La prohibition tombe naturellement, si l'on peut dire, au décès du frère aîné et le cadet peut alors hériter sans crainte. Cet interdit s'applique strictement aux frères entiers mais pas aux demi-frères ni aux frères classificatoires de lignage, ce qui explique les entorses à la règle, réelles ou supposées. Mais cette théorie n'est pas partagée par tous les Dì̀ dont beaucoup nient l'existence de sanctions surnaturelles dans ce cas. À l'inverse, un adultère entre frère aîné et femme de frère cadet est impensable; je n'en ai recueilli aucun cas et les Dì̀ avec qui j'ai discuté de ce point ont spéculé sur un tel exemple hypothétique en disant qu'il tomberait dans la catégorie des adultères, keée bèelt́, et non dans la catégorie fóó yàg fạa. Ce dernier terme est, en effet, ambigu car il désigne selon les contextes et les circonstances, les simples relations à plaisanteries ou, plus franchement, les relations sexuelles illicites avec ce type de parte- 
naires. Au contraire, le second terme indique, sans ambages, l'adultère avec tout autre femme mariée.

Aujourd'hui, la ou les veuves peuvent choisir entre plusieurs options. Si une veuve est jeune et ne veut pas être héritée par un des frères cadets de son mari défunt, elle peut rentrer chez ses parents et se chercher un nouveau mari, les enfants en bas âge qu'elle prend éventuellement avec elle étant rendus plus tard à la lignée du père. Cette désertion peut être interprétée comme une sorte de divorce qui suscite l'une ou l'autre de deux attitudes contrastées. Les lignages dì̀ ont deux politiques différentes au sujet du remboursement du prix de la fiancée : la première est de ne pas le réclamer pour rester en bons termes avec le lignage de la femme et ne pas mettre en péril une possible nouvelle alliance avec ce lignage. L'autre est de demander le remboursement, ce qui mécontente le lignage donneur et risque d'empêcher un éventuel renouvellement de l'alliance. Lorsqu'une veuve ne veut pas être héritée alors qu'il y a des partenaires potentiels, les frères du défunt peuvent réclamer le prix de la fiancée s'ils sont membres de lignages qui le demandent en cas de divorce, comme l'avait fait le neveu dans le second cas d'héritage que nous avons mentionné, mais cela semble se pratiquer de moins en moins. Dans le cas d'une veuve âgée, c'est en général son fils qui est censé s'en occuper et elle restera avec lui ; en l'absence de fils, c'est l'héritier du défunt qui la prendra en charge sans qu'il y ait remariage ou alors elle rentrera dans sa famille, ou encore ira habiter avec un membre de sa parenté proche.

\section{Les voisins}

Les Dì̀ nous donnent donc un de ces exemples que Radcliffe-Brown mentionnait mais qu'il n'a pas traité. Cependant, nous confronterons aussi les Dì̀ avec leurs voisins pour faire une comparaison régionale qui, nous semble-t-il, ancrera plus sûrement les faits dì̀ dans un contexte élargi (voir carte). Les Dì̀ font linguistiquement partie de la famille adamawa, groupe 4, qui comprend les Pèrè au sud-ouest, les Dowayo, les Duupa, les Voko et les Vere-Koma à l'ouest et au nord-ouest. Ce groupe est très proche du groupe 2, aussi voisin, comprenant les Kolbila - sur lesquels, comme pour les Voko, nous ne savons pratiquement rien - qui vivent enclavés entre les Dì̀ de dialecte gutm et naan et les Chamba-Leko qui sont un peu au-delà des Dowayo et des Koma, que les Dì̀ connaissent aussi. Au nord-est, à l'est et au sud, les Dì̀ sont entourés de populations parlant des langues du groupe 6, les Moundang à l'extrême pointe nord-est, des populations Mboum Karang et Mbéré à l'est, des Mboum au sud-est et au sud ainsi que des Nyok ou Lamé, quelquefois aussi appelés Pévé dans la littérature 
(Sachnine 1982), qui sont le groupe le plus méridional de la famille des langues tchadiques. Nous n'avons pas de renseignements utiles sur toutes ces populations mais nous pouvons raisonner sur un certain nombre d'entre elles. Nous commencerons par celles qui sont du même sous-groupe linguistique.

Chez les Pèrè, matrilinéaires ayant une terminologie de parenté comportant la règle d'obliquité crow, les termes d'adresse et de référence pour oncle et neveu sont réciproques et sont semblables à ceux des Dì̀, pạ, (Fardon 1999 : 25). Dans un manuscrit inédit, Charles-Henri Pradelles de Latour (2002: 39), précise que l'oncle est désigné comme paan walé, "paan le grand ", alors qu'Ego est, pour celui-ci, paan sénan, "paan le petit». Tout se passe comme si l'oncle maternel et le neveu utérin appartenaient à la même génération, étant séparés uniquement par une différence d'âge aîné/cadet. Dans une correspondance personnelle, le même auteur dit aussi que la femme de l'oncle est appelée yaami. Ce " terme désigne en priorité la femme du frère aîné. Il s’oppose à gomi qui désigne la femme du frère cadet. Ego peut plaisanter avec une yaami parce que la femme du frère aîné est épousable selon les lois du lévirat après la mort du frère. Ego ne plaisante pas avec une gomi, car en principe, il ne doit pas l'épouser. Cela dit, bien que la femme de l'oncle maternel soit une yaami, elle n'est pas équivalente à une belle-sœur, car elle appartient à une génération supérieure ». Dans le manuscrit inédit cité, Charles-Henri Pradelles de Latour (ibid.: 30) ajoute : «Une veuve pourrait aussi épouser un neveu utérin de son mari défunt, mais dans la pratique ce mariage n'est jamais contracté ».

Chez les Dowayo, patrilinéaires bien que se pliant à la règle d'obliquité crow qui est caractéristique des sociétés matrilinéaires, les termes pour neveu et oncle sont aussi réciproques, pabi (Barley 1983 : 6), une veuve est héritée par consentement mutuel par un frère ou un fils du défunt. La veuve peut aussi retourner chez elle après remboursement d'une partie du prix de la fiancée (ibid. : 7). La possibilité pour un neveu utérin de l'hériter n'est pas mentionnée.

Les Duupa, qui ont aussi la règle d'obliquité crow, sont patrilinéaires sauf le dernier-né qui est assigné au lignage de l'oncle maternel. Oncles et neveux s'adressent et se réferent mutuellement comme pạá, et le neveu et la femme de l'oncle s'adressent mutuellement comme époux et épouses waàá, mais le neveu n'épouse pas la veuve de l'oncle qui échoit à l'un des frères cadets du défunt. Si la veuve se remarie ailleurs, une partie au moins du prix de la fiancée doit être rendu. Le neveu est ici un "frère" de son oncle et les enfants de l'oncle sont les enfants classificatoires du neveu qui, à la mort de l'oncle, distribue l'héritage aux enfants du décédé, devenant en quelque sorte leur père (Garine 1995 : 12-21). 
Les Vere-Koma posent davantage de problèmes, car il y a plusieurs groupes sur lesquels on ne sait pas grand-chose. Françoise DumasChampion (communication personnelle) dit que chez les Koma Grmé, matrilinéaires comportant la règle d'obliquité crow, oncle maternel et neveu utérin s'appellent réciproquement ma'a mais qu'elle ignore les termes affinaux. Les Koma Ritibe (Fardon 1993 : 203) font l'équation $\mathrm{MyB}=\mathrm{eB}$ mais $\mathrm{MeB}$ est désigné par un terme spécial. On ne sait rien des termes affinaux. Il n'y a pas d'héritage de la veuve de l'oncle maternel par le neveu utérin; c'est le frère cadet du défunt qui en prend charge.

Les Vere forment un certain nombre de groupes dont la plasticité est tout à fait extraordinaire; quelques-uns sont matrilinéaires, d'autres sont franchement patrilinéaires, certains semblent avoir la double descendance unilinéaire alors que quelques groupes ont un système mixte de recrutement: un bas prix de la fiancée donne les enfants à l'oncle alors qu'un haut prix les affilie au père. Les renseignements sont particulièrement fragmentaires pour notre problème, mais les Vere de Kwoi, matrilinéaires, ont des termes non réciproques pour l'oncle et le neveu qui hérite de la femme de son oncle maternel (Meek 1931, I : 426-427). Les frères et neveux utérins des groupes de Lima et de Dongorong, matrilinéaires, héritent des veuves du défunt (ibid. : 427) sans qu'on sache trop bien les termes qui les désignent.

Les Chamba-Leko de Mapéo, qui sont à double descendance unilinéaire avec la règle d'obliquité crow, ont un terme pour oncle maternel, pobi, dont le réciproque, neveu utérin, est le même suivi d'un diminutif (Fardon 1993 : 174). L'oncle et le neveu entretiennent une relation à plaisanterie (Fardon 1988: 164). Le neveu et la femme de l'oncle sont aussi dans une relation à plaisanteries réciproque, mam.nwosi, tout comme le sont aussi les frères cadets d'un homme envers sa femme, qu'ils peuvent hériter (ibid. : 169). L'héritage de la veuve de l'oncle par le neveu est dit soit possible (ibid. : 297), soit potentiel (Fardon 1993: 182) sans que des cas d'actualisation nous soient donnés.

Si l'on passe au nord-est, on note un grand changement dans les systèmes terminologiques. Les Moundang sont patrilinéaires avec une terminologie de la parenté comportant la règle d'obliquité omaha, donc l'inverse du système terminologique de la majorité de ceux que nous avons vus jusqu'ici. Les frères aînés de la mère sont désignés comme "grandspères " alors que ses frères cadets et les neveux utérins s'appellent réciproquement nané ainsi que tous les descendants mâles de l'oncle cadet. La femme de ces oncles cadets tout comme les femmes des frères aînés sont placées dans une catégorie, nyiêné, qui inclut des relations à plaisanteries. Une veuve peut être héritée par un frère cadet du défunt ou par un neveu utérin mais, dans ce cas, seulement si sa propre mère est une sœur aînée 
entière du décédé. On retrouve ici la question du prix de la fiancée qui est un des arguments des Dì̀ pour justifier cet héritage, mais il est plus précis et restreint : puisqu'un homme ne peut théoriquement être marié que par le prix de la fiancée reçu d'une sœur aînée, ce n'est donc que le fils d'une telle femme qui peut en hériter. Mais les frères cadets de l'oncle décédé ont priorité sur le neveu par droit léviratique et, si d'aventure une veuve est héritée par le neveu - il faut en plus que l'oncle soit le frère entier de la mère de cet héritier -, les enfants reviennent aux agnats du défunt. Les relations à plaisanteries sont autorisées mais elles sont vues comme différentes: admises et même prisées entre oncle et neveu, pour lequel l'héritage est plus théorique que réel, elles sont seulement tolérées de mauvaise grâce entre frères cadets et épouse du frère aîné, pour lesquels l'héritage est possible. Si la veuve se remarie en mariage non léviratique, le nouveau mari rembourse au moins en partie le prix de la fiancée au lignage lésé (Adler 1982: 175-195).

Au sud, les Mboum, patrilinéaires, appellent l'oncle maternel náàpam. Celui-ci désigne son neveu utérin par "enfant» comme aussi tous ses autres neveux et nièces parallèles ou croisés, car le système terminologique est, en effet, très hawaïen (Hino 1978 : 51-58). Il est dit que le neveu, en l'absence de fils, hérite des propriétés et des offices de son oncle, mais rien n'est mentionné d'un héritage de sa veuve par le neveu. Au sud-est, chez les populations que les Dì̀ appellent Mboum Mbéré, ayant également une terminologie de type hawaïen, l'oncle est référé comme naamini par le neveu ; celui-ci le désigne comme gunaani ou naamini et tous deux s'appellent mutuellement naa (O'Laughlin 1973 : 398). Apparemment, il n'y a pas d'héritage de la veuve de l'oncle par le neveu (ibid.: 196-210).

Au nord-est, les Lamé patrilinéaires, que les Dì̀ appellent aussi Nyok (incluant les Pévé), ont un système terminologique hawaïen pour les cousins mais Sachnine (1982: 26) ajoute: "...la relation croisée du côté maternel est marquée terminologiquement par le terme réciproque $n d z a ̀ s i ́$ "oncle maternel/neveu (nièce) utérin(e)" ".

\section{Question d'identité}

Ce qui frappe d'abord dans cet examen, c'est la réciprocité des termes décrivant la paire oncle maternel/neveu utérin qui se répète presque comme un invariant. Les Dì̀ utilisent réciproquement, tant en termes de référence qu'en terme d'adresse, le vocable pạa, ce qui porte quelquefois à confusion car on ne sait pas toujours, lorsque deux hommes s'interpellent ainsi, qui est l'un et qui est l'autre. Les Pèrè ont aussi les mêmes termes réciproques, $p a q$, assortis d'un suffixe désignant soit l'aînesse soit la junio- 
rité générationnelle relative. Les Duupa obéissent à un patron similaire et ont aussi un terme réciproque très voisin des deux premiers en adresse et en référence, pạá.

Les Dowayo et les Chamba-Leko de Mapéo utilisent aussi des termes presque similaires pour oncle maternel, pabi pour les premiers et pobi pour les seconds. Ils sont réciproques bien qu'assortis, chez les seconds, d'un diminutif pour désigner le neveu.

Cette réciprocité est aussi le fait des Koma Grmé chez qui oncles et neveux s'appellent ma'a. Elle se répète aussi au sud-est, chez les Mboum Mbéré, où oncles et neveux se nomment réciproquement naa et se réferent comme naamini, terme employé par l'oncle et, aussi par le même terme ou alternativement comme gunaani par le neveu. Enfin, chez les Lamé ou Nyok, oncles et neveux s'appellent aussi réciproquement $n d z a ̀ s i ́$.

Que signifie cette réciprocité ? Elle se situe dans le domaine des individus qui, pourrions-nous dire, sont parents par le sang, différente de celle des affins qui peuvent se nommer réciproquement beaux-frères et bellessœurs sans qu'on sache qui est qui par rapport à la personne de référence. Elle n'est pas non plus similaire à la réciprocité des termes qui lie éventuellement des consanguins, par exemple, frères, sœurs ou encore cousins qui sont tous du même niveau générationnel. Elle est aussi différente de la réciprocité des termes grands-parents/petits-enfants ou de la semi-réciprocité qui peut lier grands-parents et petits-enfants, comme chez les Dì̀ où un grand-père appelle ses petits-enfants des deux sexes, et se réfere à ceuxci par le terme à yée, et une grand-mère par celui de à'á, les petits-enfants, garçons et filles, utilisant, quant à eux, les vocables à yéé pour le premier et $\grave{a}$ á pour la seconde. Nous avons là deux générations alternées qui sont liées par le même terme, exprimant une autre qualité d'identité que Radcliffe-Brown (1952: 69) désigne comme combinaison (combination) des générations alternées. Dans tous ces cas, cette réciprocité signifie d'abord une identité commune qui est facilement explicable en termes de positions également occupées entre les affins, aux qualités généalogiques partagées par les frères, sœurs ou cousins de même niveau générationnel, un niveau strictement horizontal, ou encore d'une identité des générations alternées opposée à la génération adjacente qui les sépare.

C'est aussi d'une identité entre oncle et neveu qu'il s'agit ici, mais différente car elle est décalée verticalement; elle concerne deux générations successives et ne s'exprime plus par des considérations positionnelles fondées sur l'identité générationnelle. Elle n'est un attribut ni de la matrilinéarité, ni de la patrilinéarité ou de la double descendance puisqu'on la retrouve, dans notre comparaison, associée à ces trois modalités. Elle est une des expressions possibles de ce que Claude Lévi-Strauss (1958) appelle 
l'«atome de parenté" constitué d'un homme et de sa sœur, du mari de celle-ci et du fils issu de cette union. Ce dernier est structuralement l'homme le plus proche du donneur de femmes et il lui est équivalent, car il le reproduit symboliquement. Cette équivalence par reproduction symbolique est même très élaborée dans nombre de mythes qui font de cette identité oncle-neveu le résultat du mariage idéal entre un frère et une sœur (Moore 1964). Cependant, cette identité ne m’a jamais été rapportée par les Dì̀ comme une des raisons de l'appropriation de la veuve de l'oncle par son neveu. Il est vrai, comme nous l'avons vu, qu'ils ont plusieurs autres théories pour l'expliquer.

Cette identité oncle-neveu est également présente ailleurs. Elle est particulièrement marquée chez les Moundang où l'identité est plus forte entre le neveu et l'oncle qui a bénéficié du prix de la fiancée de la mère du neveu puisque celui-ci est le seul a pouvoir hériter de sa veuve. Il s'agit d'abord d'une identité abstraite et idéelle qui peut être investie de contenus divers. Dans les situations matrilinéaires ou à double descendance unilinéaire, on peut même parler littéralement d'une identité de substance, n’impliquant pas toutefois nécessairement la ressemblance physique, comme le disent explicitement les Chamba-Leko, qui sont à double descendance unilinéaire. Fardon $(1999$ : 30-32 ; 1993 : 173) observe que le thème unifiant de la matrilinéarité chamba est la consubstantialité, le partage des substances impliquant, avec des gradations, tous les membres du matriclan. L'oncle maternel doit veiller à ce qui pourrait affecter le corps, la personne physique, de son neveu qui, à son tour, "has rights of privileged use of his uncle's property. Because he inherits this moveable property at his uncle's death, the two men have a common interest in such substantial properties ".

Nous n'avons pas une identité aussi étroite chez les Dì̀ où le partage des substances maternelles et paternelles sont équivalentes. La conception, hạaun terme utilisé aussi bien par les hommes que par les femmes - résulte de l'union des deux «semences" maternelles et paternelles dont les «forces" respectives au moment de la conception expliqueront les ressemblances physiques de l'enfant qui hérite, fẹe, soit du " côté " de son père soit de celui de sa mère. Que cette identité oncle/neveu s'exprime de façons diverses nous montre qu'à sa racine, c'est bien l'" atome de parenté » qui la conditionne et lui permet de se manifester sur différents registres.

Mais cette identité intergénérationnelle fait problème: le neveu est-il, ainsi que le voudrait la terminologie, un frère de l'oncle maternel plutôt qu'un neveu? Cela n'est pas toujours dit avec précision, et pour cause, car la réponse est ambivalente et quelquefois, dans la même société, fluctuante. Cependant, cette distinction aîné/cadet se trouve chez les Duupa, patrili- 
néaires, où les neveux utérins sont d'abord neveux avant de prendre la place de l'oncle après sa mort, se "remontant " ainsi d'une génération, pour procéder au partage de l'héritage entre les fils de l'oncle en s'en excluant du même coup. Le neveu devient ici un "frère " de l'oncle lors de son décès. Cette discrimination est aussi faite chez les Koma Ritibe qui distinguent le frère aîné de la mère de son neveu, mais assimilent celui-ci à un «frère » des frères cadets de sa mère. Les Dì̀ voient l'oncle plutôt comme un frère aîné puisque le neveu peut en hériter et qu'il est en compétition avec les propres frères cadets du défunt pour l'héritage de sa veuve. Mais, si l'on y regarde d'un peu plus près, on constate une gradation : l'héritage est d'abord entre frère entier de la mère et neveu, mais il est aussi permis entre deux descendants parallèles de deux sœurs issues d'une même mère; ils sont dans une relation de frères et sœurs qui s'exprime comme telle. Il devient difficile si l'oncle n'est qu'un demi-frère utérin de la mère car il n'est pas alors un vrai identique. C'est bien ici l'"atome de parenté " constitué d'un frère et de sa sœur entière, de son mari et de leur résultante, le neveu utérin, qui est premier et conditionne cet arrangement.

On retrouve cette formule, en plus élaborée, chez les Moundang. L'héritage de la veuve est la prérogative des frères cadets par droit léviratique mais le neveu, dans certaines circonstances bien particulières, peut aussi y prétendre :

- s'il est le neveu d'un frère cadet de sa mère - car il continue ici, comme identique, d'exercer ses droits sur le prix de la fiancée donné par l'oncle décédé pour obtenir sa femme en échange du prix de la fiancée donné pour la mère du neveu ;

- quand l'oncle est un frère entier de sa mère - une identité matrilatérale bien plus accentuée que celle avec un demi-frère utérin seulement. C'est ici l'identité matrilatérale et matrilinéaire la plus proche qui lui permet de revendiquer la veuve de l'oncle.

Alfred Adler (1982 : 189) nous dit que les Moundang verraient l'oncle comme un "frère femelle». Comme pour les Dì̀, c'est l'identité entre frères et sœurs entiers, ou son équivalent matrilinéaire parallèle le plus proche, qui rappelle l'identité mythique du couple frère/sœur, le couple procréateur idéal.

\section{Identités duelles}

Cette identité n'est pas toujours exprimée par l'héritage de la veuve mais elle peut l'être. C'est une condition qui semble nécessaire mais non suffisante. Cependant, elle nous montre bien que l'oncle et le neveu sont 
des identiques du côté maternel, comme le sont deux frères ou demi-frères agnatiques du côté paternel, ce qui explique la possibilité pour le neveu de se substituer à l'oncle lorsqu'il décède, ainsi qu'il le fait vis-à-vis de son frère aîné à la même occasion, à moins que cette différence des générations ne constitue une barrière à l'héritage, comme c'est le cas chez les Duupa où elle est transformée en fraternité envers le défunt, laquelle empêche l'héritage car celui-ci se fait entre générations, de père à fils, l'oncle prenant la place du père.

Cet héritage de la veuve est une variation des privilèges avunculaires que le neveu peut faire valoir. Goody (1969a) avait bien vu une partie, mais une partie seulement, du problème: dans un système patrilinéaire, et comme membre résiduel (nous dirions plutôt, quant à nous, comme membre symbolique) du lignage de son oncle, le neveu revendique des biens qui lui seraient revenus si sa mère avait été un homme. Le comportement chapardeur du neveu n'existe pas en société matrilinéaire puisque le neveu est pleinement membre du lignage de son oncle et qu'il en hérite avec, quelquefois, sa veuve comme chez les Lima et Dongorong, deux groupes vere voisins (Meek 1931, I : 427). En fait, comme Alfred Adler et Michel Cartry (1971) le signalent plus précisément, le neveu revendique une place chez son oncle. Dans les deux cas, que ce soit chapardage ou héritage de la veuve, celle-ci lui est octroyée symboliquement, marginalement et chichement. Que cette place revendiquée chez l'oncle maternel soit primordiale, c'est ce que démontre le cas des vere Gweri, Ugi, Bai et Boi qui ont deux sortes de mariage : le premier avec un prix de la fiancée très bas qui donne les enfants à l'oncle et le second, plus élevé, qui les attribue au père. Mais le prix élevé de la fiancée autorise néanmoins le neveu à envahir (invade) la maison de son oncle, surtout lorsqu'il se marie, moment où il pille son oncle qui doit aussi contribuer volontairement au mariage (Meek, 1931, I : 419). C'est donc bien le refus de l'oncle de s'attacher aussi complètement son neveu en mariage "patrilinéaire " qu’en mariage "matrilinéaire» qui engendre les relations de chapardage.

Le cas de la double descendance est particulièrement intéressant à examiner dans cette optique. Chez les Chamba-Leko, nous avons vu qu'un individu reçoit un héritage du côté paternel aussi bien que maternel. L'héritage des "moveable goods, wealth objects, money, harvested crops, and fine gowns - everything, in fact except the few objects that are commonly taken by a man's sons " passe d'oncle maternel à neveu utérin mais, malgré cet héritage et tant que l'oncle est vivant, le neveu « may appropriate small items of property, beer and fowl from his pobis [oncle maternel] compound during the latter's lifetime» (Fardon 1988: 164). Ce chapar- 
dage rituel peut être interprété comme la protestation du neveu de ne pas être l'héritier à part entière de son oncle puisqu'il n'en reçoit pas les biens immeubles (dont la maison et les terres) qui vont au fils (ibid. : 179) ${ }^{3}$.

Dans ce complexe oncle maternel/neveu utérin, il s'agit bien de la "place» du neveu face à son oncle. Les deux sont similaires, en quelque sorte des alter ego, puisqu'ils s'appellent par le même terme. Mais ils sont aussi des identiques avec leurs frères et demi-frères agnatiques dans le cas d'une succession patrilinéaire et ces deux identiques sont en concurrence. Il faut donc choisir, mais c'est presque toujours l'héritage léviratique qui sera privilégié en fait, sinon en parole.

Cette identité est toutefois ambivalente. Elle peut permettre l'héritage de la veuve comme dans certains de nos cas mais aussi l'empêcher ou, mieux, en reporter plus loin les limites. Chez les Tallensi (Fortes 1949: 277, 358-359 [index]) - l'exemple classique le mieux documenté encore aujourd'hui -, un vrai neveu ne peut hériter de la veuve de son oncle, car ils sont si proches que ce serait un inceste, même après la mort de l'oncle. En revanche, il peut hériter de la veuve du fils de son oncle, qui est un "frère " ou de celle du père de son oncle qui est un "grand-père ", sans qu'aucun exemple concret n'en soit toutefois donné. Un utérin plus éloigné peut prétendre à l'héritage mais cela est rare, car c'est l'héritage agnatique qui prime. Même si les deux façons antithétiques de manipuler l'identité se rejoignent, puisque finalement c'est l'«agnation» qui l'emporte, sa gradation est inversée : le plus proche est vu comme le premier facteur d'héritage chez les Dì̀, les Moundang et les autres voisins qui le pratiquent, alors qu'il l'interdit chez les Tallensi. Chez ces derniers, l'éloignement le favorise tandis qu'il l'amenuise chez les premiers.

MOTS CLÉS/KEYWORDS: matrilinéarité/matrilineal descent - patrilinéaritélpatrilineal descent - double descendanceldouble descent - atome de parentélatom of kinship - Dì̀ Cameroun/Cameroon.

3. Goody (1969a) avait déjà effleuré le problème de la double descendance tout en l'évitant. Les deux sociétés qu'il examine sont à double descendance, mais elles ne sont jamais nommées comme telles dans son article. Il faut attendre une contribution postérieure pour l'apprendre (Goody 1969b : 112-113). Dans la première, les LoWiili, rien n'est hérité du côté matrilinéaire et le chapardage rituel est pleinement assumé. Dans la seconde, les LoDagaba, l'héritage des biens mobiliers s'effectue matrilinéairement et le chapardage rituel « is so played down [...] that it is virtually non-existent...» (Goody 1969a : 68). Le problème est que, malgré les droits d'héritage, ce chapardage est toujours là, comme chez les ChambaLeko, et ce n'est pas l'absence de ces droits qui peut pleinement l'expliquer. Il n'est pas question de nier que l'héritage joue un rôle important dans cette affaire, mais quand sa présence laisse tout de même place à un chapardage, fût-il virtuel, on est en droit d'affirmer qu'il tire son origine de quelque chose de plus fondamental. 
Adler, Alfred

1982 La Mort est le masque du roi. La royauté sacrée chez les Moundang du Tchad. Paris, Payot («Bibliothèque scientifique»).

\section{Adler, Alfred \& Michel Cartry}

1971 "La transgression et sa dérision", L'Homme 11 (3) : 5-63.

\section{Barley, Nigel}

1983 Symbolic Structures. An Exploration of the Culture of the Dowayos. Cambridge, Cambridge University Press/Paris, Éd. de la Maison des sciences de l'homme.

\section{Fardon, Richard}

1988 Raiders and Refugees. Trends in Chamba Political Development, 1750-1950. Washington, Smithsonian Institution Press ("Smithsonian Series in Ethnographic Enquiries ").

1993 "Alliance et ethnicité. Un système régional de l'Adamawa ", in Françoise Héritier-Augé \& Élisabeth Copet-Rougier, Les Complexités de l'alliance. III. Économie, politique et fondements symboliques (Afrique). Paris, Éditions des Archives contemporaines ("Ordres sociaux ») : 165-210.

1999 «Pere and Chamba : A Report on Comparative Researches in Adamawa, North Cameroon ", Ngaoundéré-Anthropos IV : 5-52.

\section{Fortes, Meyer}

1949 The Web of Kinship among the Tallensi. The Second Part of an Analysis of the Social Structure of a Trans-Volta Tribe. London, Oxford University Press for International African Institute.

Garine, Éric de

1995 Le Mil et la bière. Le système agraire des Duupa du massif de Poli (NordCameroun). Nanterre, Université de Paris X.

\section{Goody, Jack}

1969a "The Mother's Brother and the Sister's Son in West Africa " (1959), in Jack
Goody, Comparative Studies in Kinship.

London, Routledge \& Kegan Paul : 39-90.

1969b "The Classification of Double

Descent Systems » (1961), in Comparative

Studies... : 91-119.

Héritier, Françoise

1994 Les Deux sours et leur mère.

Anthropologie de l'inceste. Paris, Odile Jacob.

Hino, Shun'ya

1978 The Classified Vocabulary of the

Mbum Language of Mbang Mboum - with

Ethnographical Descriptions. Tokyo, ILCAA.

\section{Lévi-Strauss, Claude}

1958 «L'analyse structurale en linguistique et en anthropologie ", in Anthropologie structurale. Paris, Plon : 37-62.

\section{Meek, Charles Kingsley}

1931 Tribal Studies in Northern Nigeria, vol. I. London, Kegan Paul, Trench, Trubner and Co.

\section{Moore, Sally Falk}

1964 "Descent and Symbolic Filiation", American Anthropologist 66 (6) : 1308-1320.

Muller, Jean-Claude

1997 «Un système crow patrilinéaire : les Dì̀ de Mbé (Adamaoua, Nord-Cameroun). Essai de triangulation méthodologique", Anthropologie et Sociétés 21 (2-3) : 125-141. 1998 «Le mariage chez les Dì̀ de Mbé (Adamaoua, Cameroun). Un système semicomplexe inhabituel », L'Homme 148 : 47-78.

\section{Murdock, George Peter}

1949 Social Structure. Glencoe, The Free Press.

\section{O'Laughlin, M. Bridget}

1973 Mbum Beer Parties: Structures of Production and Exchange in an African Social Formation. Ann Arbor, University Microfilms (Ph. D. thesis). 
Pradelles de Latour, Charles-Henry

2002 Le Gerèm des Pèrè. Monographie d'une société matrilinéaire du Cameroun. Ms.

\section{Radcliffe-Brown, Alfred Reginald}

1952 "The Study of Kinship Systems " (1941), in Radcliffe-Brown, Structure and Function in Primitive Society. New York, The Free Press : 49-89.
Sachnine, Michka

1982 Le lamé (Nord-Cameroun). Paris, SELAF/ACTT ("Langues et cultures africaines " 1).

Seignobos, Christian

1987 Le poney du Logone. Maison-Alfort, IEMVT ("Études et synthèses» 23).

RÉSUMÉ/ABSTRACT

Jean-Claude Muller, Question d'identité. L'héritage de la veuve de l'oncle maternel chez les Diì (Nord-Cameroun). - Cet article reprend un vieux problème examiné par Radcliffe-Brown, mais jamais complètement résolu: celui de l'héritage de la veuve de l'oncle maternel, qui est attesté dans des sociétés les plus diverses, en concurrence avec d'autres types d'héritage. Leurs logiques respectives sont analysées chez les Dì̀ de l'Adamaoua et les voisins immédiats qu'ils connaissent. Cette dialectique entre les héritages contrastés repose sur une quasi-identité de substance entre l'oncle et le neveu et une autre identité, mais différente, liant les «frères» agnatiques - identités entre lesquelles il faut trancher.
Jean-Claude Muller, Question of Identity. The Inheritance of the Maternal Uncle's Widow among the Dii (North Cameroon). - This article resurrects an old problem evoqued by Radcliffe-Brown which he left unresolved: the inheritance of the maternal uncle's wife by his uterine nephew. This inheritance is found in many diverse societies, concurrently with other types of inheritance. These respective inheriting logics are here analysed among the Diì of Adamawa and the neighbors they know. These dialectical choices between the contrasted heritages are grounded on a quasi identity between uncle and nephew and another identity, albeit different, linking the agnatic "brothers ». The choice must be between one or the other. 